\title{
Estudo de caso sobre a implementação de técnicas de blindagem em servidores Linux baseada na detecção de vulnerabilidades e tentativas de intrusão
}

\author{
Álisson Wegner Sousa ${ }^{1}$, Gabriel Marvel Vaz ${ }^{1}$ Vinícius Röpke $^{1}$, Lucas Vargas Dias ${ }^{1}$, \\ Tiago Antonio Rizzetti ${ }^{1}$
}

\author{
${ }^{1}$ Redes de Computadores - Universidade Federal de Santa Maria (UFSM) \\ Caixa Postal 5082 -- CEP: 97.105-900 - Santa Maria - RS - Brazil \\ \{alissonwegner,gabrielmarvelvaz,viniropke, lucas_dias,rizzetti\} \\ aredes.ufsm.br
}

\begin{abstract}
Resumo. Neste artigo é apresentado um estudo de caso baseado na detecção de vulnerabilidades e tentativas de intrusão em uma infraestrutura de rede composta por servidores Linux, seguido do emprego de técnicas de blindagem para mitigar as ameaças existentes. Para realizar as detecções foram utilizados o scanner OpenVAS e o Intrusion Detection System (IDS) Suricata. Com a implementação das técnicas de blindagem foi possível tornar a rede mais segura, mitigando as vulnerabilidades existentes e tentativas de intrusão. Ademais, concluiu-se que o processo de identificar, compreender, solucionar e testar as soluções dessas ameaças pode ser custoso em relação ao tempo empregado. Devido a isso, há necessidade de maior automação para que o tratamento de questões relacionadas à segurança das organizações e indivíduos se torne mais rápido e eficiente.
\end{abstract}

\section{Introdução}

Em um ambiente amplamente conectado, novas vulnerabilidades são descobertas diariamente e os riscos aumentam de forma expressiva, tornando imprescindível e imediata a necessidade de empregar técnicas e ferramentas de segurança para mitigá-las. Scanners de vulnerabilidades e Intrusion Detection Systems (IDSs) podem auxiliar no processo de avaliação de riscos, permitindo identificar vulnerabilidades e ameaças, que são etapas importantes neste processo [William Stallings 2014]. Hardening trata-se de proteger um sistema através da redução de suas possíveis vulnerabilidades, por meio de configurações que implementam controles específicos [Melo 2014]. De acordo com as técnicas apresentadas por [Melo 2014], para se realizar o hardening, pode ser utilizada a abordagem de proteção de perímetro, que é executada diretamente no firewall, protegendo a rede em geral. Em conjunto, pode ser empregada uma abordagem individualizada, visando tratar especificidades de determinados equipamentos, aumentando sua segurança.

Este trabalho tem como objetivo apresentar um estudo de caso desenvolvido na rede de uma instituição de ensino, no qual foram utilizadas ferramentas para detecção de vulnerabilidades e intrusões. Com base nos dados obtidos, definiu-se um fluxo de avaliação e mitigação das vulnerabilidades, seguido do emprego de técnicas de filtragem e blindagem necessárias para tornar a rede mais segura. A partir das medidas implementadas, foi possível observar o impacto destas, e ainda, alguns desafios a serem enfrentados no âmbito de segurança da rede. 


\section{Trabalhos Relacionados}

No trabalho proposto por [Chalvatzis et al. 2019] é realizado um comparativo entre as ferramentas de escaneamento de vulnerabilidades Nessus, OpenVAS e Nmap Scripting Engine (NSE). Os autores avaliam questões como capacidade de detecção de vulnerabilidades, velocidade e usabilidade. O Nessus apresenta pequena vantagem nessa comparação, principalmente devido à sua base de conhecimento, que abrange um número maior de Common Vulnerabilities and Exposuress (CVE). Porém, considerando que algumas CVEs não são detectadas por serem muito antigas ou específicas, essa diferença pode ser muito menor do que parece.

Em [Huang et al. 2012] optou-se pelo IDS Snort devido à sua capacidade de monitorar e analisar o fluxo de dados em tempo real, gerando alertas conforme as regras configuradas [Snort 2020]. A partir da análise dos alertas obtidos, foram selecionadas técnicas de hardening para aumentar a segurança da rede através de configurações no $f i$ rewall. Com isso, foi possível observar os fluxos de tráfego e resolver problemas como o volume de big data e a baixa velocidade de detecção de vulnerabilidades, consequentemente melhorando o desempenho da rede.

Já em [Mello 2017], o autor realiza a implementação de alguns mecanismos de blindagem em um servidor web de uma instituição de saúde, empregando técnicas baseadas no princípio de privilégio mínimo, além de configurações específicas nas aplicações Apache e MySQL. Após as blindagens, o autor utiliza a ferramenta Lynis para buscar vulnerabilidades, de modo a verificar a eficácia das técnicas empregadas.

\section{Estudo de caso}

A rede sob a qual foi realizado o estudo de caso possui uma faixa de endereços Internet Protocol versão 4 (IPv4) públicos, ou seja, acessíveis pela Internet. Portanto, os principais hosts da rede, entre eles servidores Domain Name System (DNS), Dynamic Host Configuration Protocol (DHCP) e servidores Web, são acessíveis externamente. Devido a isso, o trabalho foi focado nestes hosts. O fluxograma da Figura 1 apresenta a ordem lógica das ações tomadas a partir da detecção das ameaças, que serviu para otimizar o processo de blindagem e pode ser utilizada em outros ambientes para este propósito.

A estrutura desta Seção é composta pelo mapeamento das vulnerabilidades existentes através da ferramenta OpenVAS e correção das falhas encontradas, descrito na Subseção 3.1. Em sequência, foram tratadas a detecção de tentativas de intrusão com o IDS Suricata e mitigação das respectivas ameaças na Subseção 3.2. Por fim, na Subseção 3.3, foi realizada uma análise da eficácia das soluções implementadas através de um novo escaneamento e também de uma nova amostra de tentativas de intrusão.

\subsection{Escaneamento de vulnerabilidades com OpenVAS e técnicas de blindagem empregadas}

A ferramenta OpenVAS trata-se de um software livre para escaneamento de vulnerabilidades acompanhado por um feed da comunidade Greenbone que inclui mais de 50.000 testes de vulnerabilidade [OpenVAS 2020]. A varredura pode ser configurada e executada via interface $W e b$, na qual também é possível visualizar os resultados e gerar relatórios a partir destes. Vale ressaltar que a faixa de endereços públicos utilizada possui uma 


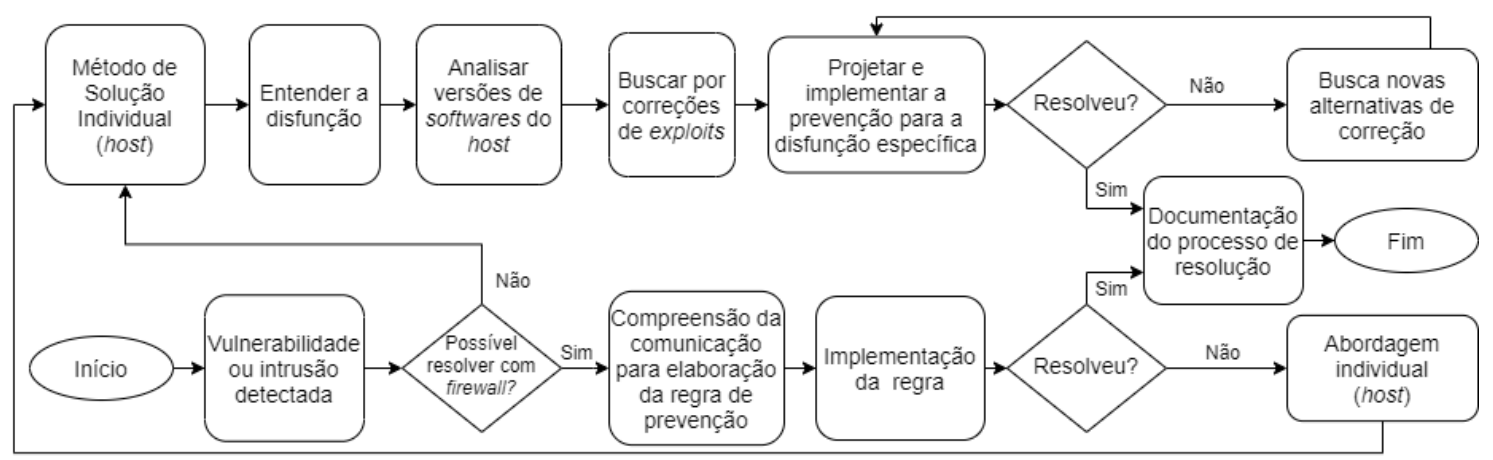

Figura 1. Metodologia adotada para mitigação das ameaças.

máscara de rede $/ 25$ e em torno de 10 hosts ativos. O escaneamento encontrou 27 vulnerabilidades. Foi encontrada apenas uma vulnerabilidade de severidade alta, 18 de nível médio e 8 de nível baixo. De acordo com as recomendações do NIST (National Institute of Standards and Technology) [Stoneburner et al. 2002], as probabilidades de uma possível vulnerabilidade ser explorada por uma determinada fonte de ameaça pode ser descrita como alta, média ou baixa.

Na Tabela 1 temos a listagem das vulnerabilidades encontradas, bem como seu nível de severidade e descrição. Foram encontradas 27 vulnerabilidades no total. Porém algumas detecções são redundantes, ou seja, as mesmas vulnerabilidades foram encontradas em hosts distintos. No total, foram encontradas 10 vulnerabilidades distintas.

Tabela 1. Resultados do escaneamento com o OpenVAS.

\begin{tabular}{|c|c|c|}
\hline Nome da vulnerabilidade & Severidade & Descrição \\
\hline OS End Of Life Detection & Alta & Sistema operacional desatualizado. \\
\hline $\begin{array}{c}\text { HTTP Debbuging Methods (TRACE/TRACK) } \\
\text { Enabled }\end{array}$ & Média & $\begin{array}{l}\text { Método TRACE ativado, vulnerável a } \\
\text { ataques Croos-Site-Tracing (XST). }\end{array}$ \\
\hline Unprotected Web App Installers (HTTP) & Média & $\begin{array}{l}\text { Páginas de configuração de aplicativos } \\
\text { disponíveis para acesso no servidor Web. }\end{array}$ \\
\hline Missing 'httpOnly' Cookie Attribute & Média & $\begin{array}{l}\text { Cookies não estão usando o atributo de } \\
\text { cookie seguro 'httponly'. }\end{array}$ \\
\hline Kill service with random data & Média & $\begin{array}{l}\text { Um atacante pode executar código } \\
\text { arbitrário e travar o sistema. }\end{array}$ \\
\hline $\begin{array}{c}\text { Cleartext Transmission of Sensitive Information } \\
\text { via HTTP }\end{array}$ & Média & $\begin{array}{l}\text { Uso de HTTP, que não utiliza } \\
\text { criptografia na comunicação. }\end{array}$ \\
\hline Telnet Unencrypted Cleartext Login & Média & $\begin{array}{l}\text { Uso de Telnet, que não utiliza } \\
\text { criptografia na comunicação }\end{array}$ \\
\hline SSH Weak Encryption Algorithms Supported & Média & $\begin{array}{l}\text { Servidor SSH utilizando algoritmos de } \\
\text { criptografia fracos, como: "3-des-cbc", } \\
\text { "aes128-cbc" entre outros. }\end{array}$ \\
\hline jQuery < 1.9.0 XSS Vulnerability & Média & $\begin{array}{l}\text { Versão do jQuery vulnerável a ataques } \\
\text { Cross-site Scripting (XSS). }\end{array}$ \\
\hline TCP Timestamps & Baixa & $\begin{array}{l}\text { Permite que um atacante obtenha o } \\
\text { uptime do servidor remotamente. }\end{array}$ \\
\hline
\end{tabular}

Identificadas e compreendidas as vulnerabilidades, foram realizadas as configurações necessárias para sanar os problemas existentes. As técnicas empregadas consistiram 
em configurações específicas nos servidores, que são descritas resumidamente na Tabela 2.

Tabela 2. Técnicas implementadas para mitigar as vulnerabilidades.

\begin{tabular}{|c|c|}
\hline Vulnerabilidade & Solução implementada \\
\hline OS End Of Life Detection & \multirow{2}{*}{ Atualização do sistema operacional. } \\
\hline Kill service with random data & \\
\hline $\begin{array}{c}\text { Cleartext Transmission of Sensitive } \\
\text { Information via HTTP }\end{array}$ & Migração das páginas Web de HTTP para HTTPS. \\
\hline $\begin{array}{l}\text { HTTP Debugging Methods } \\
\text { (TRACE/TRACK) Enabled }\end{array}$ & Desativação do método TRACE no servidor Web. \\
\hline Unprotected Web App Installers (HTTP) & $\begin{array}{l}\text { Modificação nas permissões de acesso às páginas de } \\
\text { configuração e instalação do servidor Web. }\end{array}$ \\
\hline Missing “httpOnly" Cookie Attribute & $\begin{array}{c}\text { Ativação do atributo de cookie seguro "httponly" no } \\
\text { servidor Web. }\end{array}$ \\
\hline Relacionadas à Telnet e SSH & Notificação dos administradores responsáveis. \\
\hline TCP timestamps & Desativação da extensão "tcptimestamps". \\
\hline
\end{tabular}

\subsection{Detecção de intrusões com o IDS Suricata e técnicas de blindagem empregadas}

Optou-se por posicionar o IDS Suricata de forma passiva na rede, analisando os pacotes encaminhados e recebidos, objetivando detectar a existência de intrusos. Na figura 2 vemos a localização do IDS na rede, que recebe uma cópia do tráfego através do mecanismo de port mirroring disponível nos switches.

O Suricata foi escolhido pelo seu mecanismo multithread, que fornece maior velocidade na análise do tráfego, usando um poder maior de processamento fornecido pelos processadores multi-núcleo [Wong et al. 2017].

A ferramenta disponibiliza um conjunto de regras padrão da comunidade [Suricata 2019], o qual foi utilizado neste estudo de caso. Após 5 dias de execução (anteriores às blindagens), foram gerados 69.990 alertas. O gráfico apresentado na Figura 3 mostra a porcentagem equivalente aos principais tipos de assinaturas detectadas. A partir do relatório de detecção foi possível identificar que a maioria das tentativas de intrusão relacionavam-se à scans, tentativas de acesso remoto via SSH ou exploração de exploits em servidores Web.

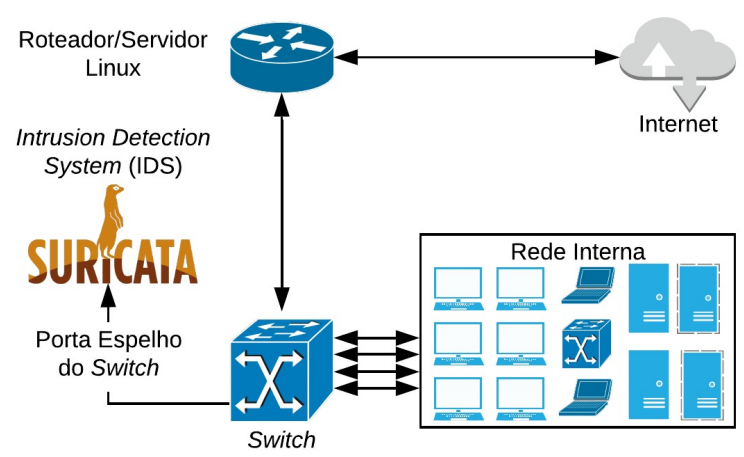

Figura 2. Localização do IDS. 


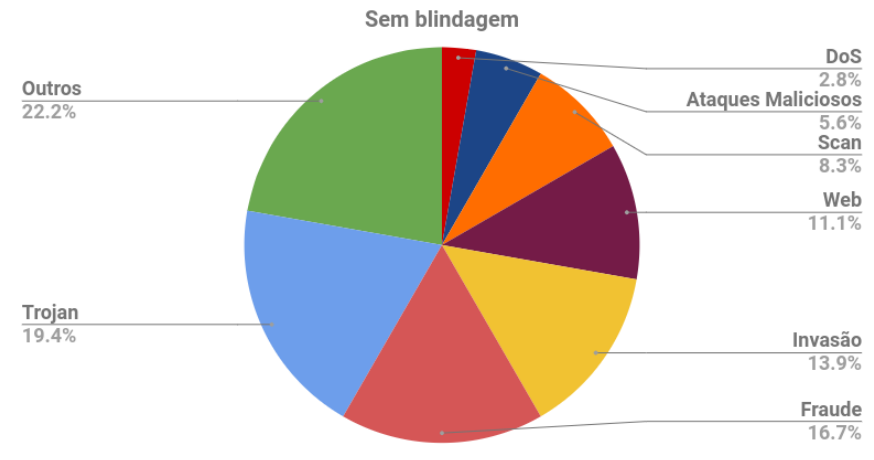

Figura 3. Porcentagem de assinaturas detectadas.

Com o objetivo de mitigar as ameaças detectadas, foram utilizadas as técnicas descritas na Tabela 3, baseando-se nos processos de hardening propostos em [Melo 2014] de acordo com cada ameaça. Após a implementação dos mecanismos de blindagem, foram empregados os mesmos processos de detecção, que serão descritos na Seção 3.3, desta vez com o objetivo de verificar a eficácia da mitigação das ameaças.

Tabela 3. Técnicas implementadas com base nas detecções do IDS.

\begin{tabular}{|l|l|}
\hline Ameaça & Medidas de Prevenção \\
\hline Scan & - \\
\hline Tentativas SSH & Alteração da porta padrão SSH em todos os servidores que possuem IP público \\
\hline Exploits Web & $\begin{array}{c}\text { Bloqueio de portas e páginas Web que não necessitam ser acessadas } \\
\text { publicamente; }\end{array}$ \\
Implementação do HTTPS nos servidores Web.
\end{tabular}

\subsection{Resultados}

Conforme apresentado na Figura 4, o novo escaneamento do OpenVAS detectou apenas sete vulnerabilidades, sendo quatro delas referentes a hosts fora do domínio dos autores. Das outras três vulnerabilidades encontradas, uma delas é referente à versão do jQuery, que ainda não foi resolvida também por questões externas ao domínio dos autores e as outras duas são referentes aos servidores $W e b$, nos quais serão feitas as configurações necessárias para mitigá-las. Com o novo escaneamento observou-se uma redução de 89,95\% no número de vulnerabilidades encontradas.

Já com o Suricata, após 5 dias de análise posteriores às blindagens foram gerados 20.373 alertas, o que equivale a uma diminuição de $30 \%$ em relação às detecções iniciais. No gráfico da Figura 5 podemos observar que as tentativas de escaneamento predominaram neste período, seguidas de tentativas de ataques maliciosos e demais intrusões variadas.

\section{Comparativo entre a proposta e os trabalhos relacionados}

Esta Seção apresenta a comparação dos trabalhos relacionados com o estudo de caso descrito neste artigo, conforme ilustrado na Tabela 4. O comparativo é feito baseado nos dois pontos principais abordados neste estudo de caso, bem como os resultados obtidos. 


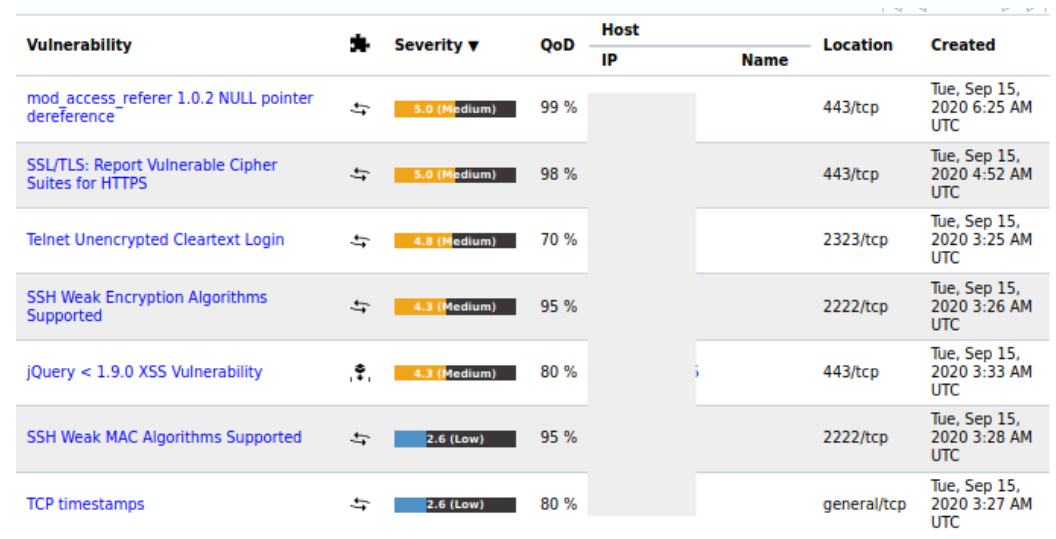

Figura 4. Resultado do escaneamento com o OpenVAS após as blindagens.

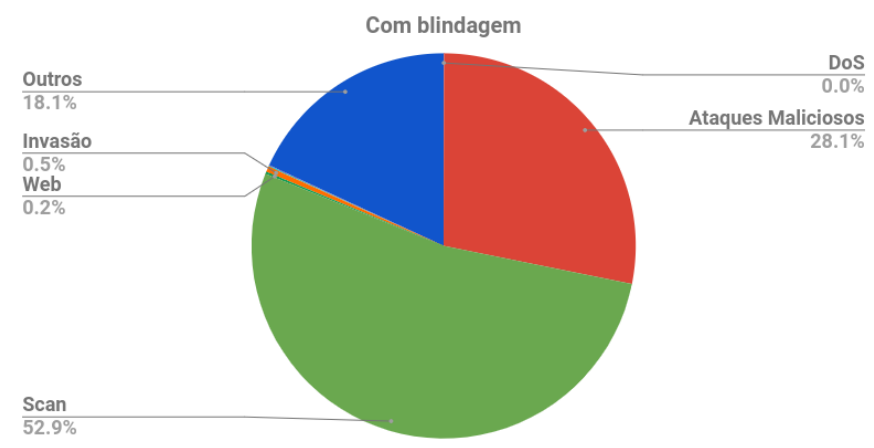

Figura 5. Porcentagem de assinaturas detectadas após a blindagem.

\section{Conclusões e trabalhos futuros}

Com a implementação do trabalho foi possível detectar ameaças e mitigá-las, tornando a rede consideravelmente mais segura e confiável para seus usuários. Além disso, os procedimentos de segurança realizados foram padronizados através da documentação, visando tornar a detecção e prevenção de ameaças mais usual aos administradores, possibilitando controlar a rede de uma forma segura.

Mesmo com a documentação e padronização dos passos a serem realizados, a tarefa de detecção de vulnerabilidades e intrusões, bem como a implementação de técnicas de blindagem, apresenta-se consideravelmente custosa em termos de complexidade e tempo demandado. Haja vista, se identifica a necessidade de automatizar estes processos através de um software capaz de realizar as blindagens automaticamente de acordo com a detecção de intrusão ou vulnerabilidade inserida. O desenvolvimento deste software será estudado para implementação em trabalhos futuros.

\section{Referências}

Chalvatzis, I., Karras, D. A., and Papademetriou, R. C. (2019). Evaluation of security vulnerability scanners for small and medium enterprises business networks resilience towards risk assessment. In 2019 IEEE International Conference on Artificial Intelligence and Computer Applications (ICAICA), pages 52-58. 
Tabela 4. Comparativo com os trabalhos relacionados.

\begin{tabular}{|c|l|l|l|}
\hline Trabalho & \multicolumn{1}{|c|}{ Detecção } & \multicolumn{1}{|c|}{ Blindagem } & \multicolumn{1}{c|}{ Resultados } \\
\hline [Chalvatzis et al. 2019] & $\begin{array}{l}\text { Os autores realizam um } \\
\text { comparativo entre os } \\
\text { scanners Nessus, NSE e } \\
\text { OpenVAS. }\end{array}$ & $\begin{array}{l}\text { O trabalho não abrange } \\
\text { este ponto. }\end{array}$ & $\begin{array}{l}\text { Nessus e OpenVAS apre- } \\
\text { sentaram vantagem em re- } \\
\text { lação ao NSE de acordo } \\
\text { com as métricas utilizadas, } \\
\text { com o Nessus levando pe- } \\
\text { quena vantagem no geral. }\end{array}$ \\
\hline [Huang et al. 2012] & $\begin{array}{l}\text { Utiliza o IDS Snort para } \\
\text { detectar intrusões. }\end{array}$ & $\begin{array}{l}\text { Realiza configurações no no } \\
\text { firewall de acordo com as } \\
\text { detecções. }\end{array}$ & $\begin{array}{l}\text { Foram resolvidos proble- } \\
\text { mas como volume de big } \\
\text { data e baixa velocidade de } \\
\text { detecção de intrusões na } \\
\text { rede. }\end{array}$ \\
\hline [Mello 2017] & $\begin{array}{l}\text { Utiliza a ferramenta Ly- } \\
\text { nis para detectar vulne- } \\
\text { rabilidades após imple- } \\
\text { mentar as técnicas de } \\
\text { blindagem. }\end{array}$ & $\begin{array}{l}\text { Implementa mecanismos } \\
\text { como limitação de horário } \\
\text { para acesso remoto, confi- } \\
\text { guração do comando sudo, } \\
\text { suid bit, entre outras técni- } \\
\text { cas baseadas no princípio } \\
\text { do privilégio mínimo. }\end{array}$ & $\begin{array}{l}\text { Com as técnicas imple- } \\
\text { mentadas foi possível ele- } \\
\text { var o nível de segurança do } \\
\text { servidor Web, tornando-o } \\
\text { menos propenso a ataques } \\
\text { danosos. }\end{array}$ \\
\hline [Autores 2020] & $\begin{array}{l}\text { Uso do scanner Open- } \\
\text { VAS para detecção de } \\
\text { vulnerabilidades especí- } \\
\text { ficas nos servidores, em } \\
\text { conjunto com o IDS Su- } \\
\text { ricata para detectar tenta- } \\
\text { tivas de intrusão à rede. }\end{array}$ & $\begin{array}{l}\text { Implementa individu- } \\
\text { almente as técnicas } \\
\text { necessárias para soluci- } \\
\text { onar as vulnerabilidades } \\
\text { existentes e também con- } \\
\text { figurações no firewall para } \\
\text { tornar a rede mais segura. }\end{array}$ & $\begin{array}{l}\text { Aumento da segurança dos } \\
\text { principais servidores e da } \\
\text { rede em geral a partir das } \\
\text { técnicas implementadas. E } \\
\text { além disso, diminuição no } \\
\text { número de tentativas de in- } \\
\text { trusão. }\end{array}$ \\
\hline
\end{tabular}

Huang, C., Xiong, J., and Peng, Z. (2012). Applied research on snort intrusion detection model in the campus network. In 2012 IEEE Symposium on Robotics and Applications (ISRA), pages 596-599.

Mello, B. F. (2017). Estudo da aplicação da técnica de Hardening nos servidores web do Hospital de Clínicas de Porto Alegre. Trabalho de Conclusão de Curso - Gestão da Segurança da Informação - Unisul Virtual.

Melo, S. (2014). Hardening em Linux. Escola Superior de Redes - RNP.

OpenVAS (2020). OpenVAS - Open Vulnerability Assessment Scanner.

Snort (2020). Snort Overview.

Stoneburner, G., Goguen, A., and Alexis, F. (2002). Risk Management Guide for Information Technology Systems. Recommendations of the National Institute of Standards and Technology, NIST Special Publication 800-30.

Suricata (2019). Rule Management with Suricata-Update.

William Stallings, L. B. (2014). Segurança de Computadores: Princípios e Práticas. Rio de Janeiro.

Wong, K., Dillabaugh, C., Seddigh, N., and Nandy, B. (2017). Enhancing suricata intrusion detection system for cyber security in scada networks. In 2017 IEEE 30th Canadian Conference on Electrical and Computer Engineering (CCECE), pages 1-5. 